\title{
Removal of temporary cements following an immediate dentin hybridization approach: a comparison of mechanical and chemical methods for substrate cleaning
}

\author{
Augusti, Davide ; Re, Dino ; Özcan, Mutlu ; Augusti, Gabriele
}

\begin{abstract}
This study evaluated the microshear bond strength ( SBS) of a composite resin cement to a pre-hybridized dentinal substrate exposed to two kinds of temporary materials; the influence of different cleaning techniques was investigated. Dentinal surfaces were conditioned with an etch-and-rinse adhesive system to obtain an immediately-sealed (IDS) layer. Each surface was divided into quadrants and covered (1) with an eugenol-free (NE_Group) or (2) with a resin-based provisional agent (CL_Group). After storage, the temporary cement was removed by using one of the following methods: (1) Hand-scaler [S]; (2) Alumina air-abrasion [SB]; (3) Glycine-powder air-abrasion [Gly]; (4) D-Limonene chemical solvent [Or]. A new IDS layer was then created; polyethylene tubes were placed on dentin surfaces and filled with a dual-cure resin cement. A universal testing machine was used for the SBS tests; conditioned surfaces were analyzed at SEM. Means and SD were calculated; a two-way ANOVA (a: 0.05) was performed to detect significant differences among groups. For NE groups, mean SBS values (MPa) were: $21.6 \pm$ $6.6\left[\mathrm{NE} \_\mathrm{NoT}\right] ; 20.7 \pm 4.5\left[\mathrm{NE} \_\mathrm{Or}\right] ; 20.1 \pm 6.6\left[\mathrm{NE \_ SB}\right] ; 19.1 \pm 5.3\left[\mathrm{NE} \_\mathrm{S}\right] ; 17.8 \pm 2.2\left[\mathrm{NE} \_\mathrm{Gly}\right]$. No significant differences were found among tested treatments within NE (p: 0.5493). For CL groups, mean SBS values $(\mathrm{MPa})$ ranged from $15.8 \pm 2.8$ (CL_S50) to $19.4 \pm 2.9$ (CL_Gly). Cleaning of the substrate with glycine air-abrasion statistically improved SBS values with respect to aluminum-oxide (CL_SB: $15.8 \pm 2.8$ ) or scaling (CL_S: $16.0 \pm 2.4$ ). The application of the IDS technique was effective for preservation of freshly-cut dentin from adverse effects of temporary materials. Glycine air-abrasion is suggested when a temporary resin cement is adopted.
\end{abstract}

DOI: https://doi.org/10.1080/01694243.2017.1381015

Posted at the Zurich Open Repository and Archive, University of Zurich

ZORA URL: https://doi.org/10.5167/uzh-162812

Journal Article

Accepted Version

Originally published at:

Augusti, Davide; Re, Dino; Özcan, Mutlu; Augusti, Gabriele (2018). Removal of temporary cements following an immediate dentin hybridization approach: a comparison of mechanical and chemical methods for substrate cleaning. Journal of Adhesion Science and Technology, 32(7):693-704.

DOI: https://doi.org/10.1080/01694243.2017.1381015 


\section{UNIVERSITÀ DEGLI STUDI DI MILANO \\ DIPARTIMENTO DI SCIENZE BIOMEDICHE, CHIRURGICHE E ODONTOIATRICHE}

Manuscript Title Page:

Removal of temporary cements following an immediate dentin hybridization approach: a comparison of mechanical and chemical methods for substrate cleaning.

Article Type:

Original scientific article

Authors Order (Name/Surname):

Davide Augusti *

Dino $\operatorname{Re}$ *

Mutlu Özcan **

Gabriele Augusti * davide.augusti@unimi.it

dino.re@unimi.it

mutlu.ozcan@zzm.uzh.ch

g.augusti@libero.it

* Division of Oral Rehabilitation, Istituto Stomatologico Italiano, University of Milan, Italy

** Center of Dental Medicine, University of Zurich

\section{Corresponding Author \\ Gabriele Augusti \\ E-mail: \\ g.augusti@libero.i†}

Istituto Stomatologico Italiano, University of Milan, Via Pace 21, 20122 Milan, Italy 


\section{Extended Abstract}

The aim of this study was to evaluate the microshear bond strength of a composite resin cement to a pre-hybridized dentinal substrate exposed to two kinds of temporary materials; the influence of several chemo-mechanical cleaning techniques on the adhesion to previously sealed surfaces was investigated.

Dentin surfaces of 24 human molars were exposed and conditioned with a 3-step, etch-andrinse adhesive system for development of an immediate dentinal sealed (IDS) layer. The IDS layer on each tooth was divided into four quadrants; one of them was used as a control surface (NoT). The teeth were subsequently divided according to the type of temporary material used for contamination of the remaining three quadrants: 14 teeth were allocated to the group (NE Group) where an eugenol-free temporary cement (TempBond NE®) was used; the remaining 10 teeth where addressed for procedures involving a resin-based temporary agent (TempBond Clear $\left.{ }^{\circledR}\right)$ (CL Group). Dentin surfaces were coated with the provisional cement and stored in distilled water at $37^{\circ} \mathrm{C}$ for $24 \mathrm{~h}$. After storage, the temporary cement layer was removed from

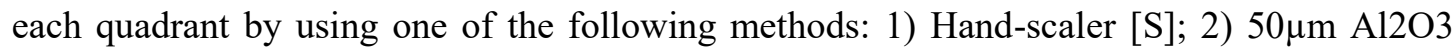
air-abrasion [SB]; 3) $25 \mu \mathrm{m}$ glycine powder air-abrasion [Gly]; 4) D-Limonene chemical solvent [Or] (only for NE Group). A new IDS layer was then created; polyethylene tubes (inner area: $1 \mathrm{~mm} 2$ ) were placed on dentin surfaces and filled with a dual-cure resin cement. The luting agent was light cured for 40s; a total of 4 bonded specimens were obtained for each tooth. A universal testing machine was used for the microshear bond strength ( $\mu \mathrm{SBS}$ ) tests; additional samples were analyzed at SEM for visualization of conditioned surfaces. Means and SD obtained from $\mu$ SBS tests were calculated; A two-way analysis of variance (two-way ANOVA; a: 0,05) was carried out to detect differences among study groups.

For NE group, mean $\mu \mathrm{SBS}$ values (MPa) were: $21.6 \pm 6.6$ [NE_NoT]; $20.7 \pm 4.5$ [NE_Or]; $20.1 \pm 6.6$ [NE_SB]; $19.1 \pm 5.3$ [NE_S]; $17.8 \pm 2.2$ [NE_Gly]. No significant differences were found among tested treatments within NE (p: 0,5493). For CL group, mean $\mu$ SBS values (MPa) ranged from $15.8 \pm 2.8$ (CL_S50) to $19.4 \pm 2.9$ (CL_Gly). A significant difference was found among study groups within CL (p: 0,0188): cleaning of the substrate with glycine airabrasion statistically improved $\mu$ SBS values with respect to aluminum-oxide sandblasting (CL_SB: $15.8 \pm 2.8)$ or scaling $\left(C L \_S: 16.0 \pm 2.4\right)$.

The application of the immediate dentin sealing (IDS) technique, in association with one of the tested cleaning methods, was effective for preservation of freshly-cut dentin from adverse effects of temporary materials. Best $\mu$ SBS values were identified for specific temporary luting-agent/surface treatment combinations. The use of glycine powder air-abrasion is suggested when a temporary resin cement is adopted.

Keywords: Immediate Dentin Sealing; Temporary cement; Surface treatment; Adhesive luting; Micro-shear bond strength. 


\section{Short (250-words) Abstract}

This study evaluated the microshear bond strength of a composite resin cement to a pre-hybridized dentinal substrate exposed to two kinds of temporary materials; the influence of different cleaning techniques was also investigated.

Dentinal surfaces of human molars were conditioned with an etch-and-rinse adhesive system to obtain an immediately-sealed (IDS) layer. Each tooth was divided into quadrants and covered 1) with an eugenol-free (NE_Group) or 2) with a resin-based provisional agent (CL_Group). After storage, the temporary cement was removed by using one of the following methods: 1)Hand-scaler [S]; 2)Alumina air-abrasion [SB]; 3)Glycine-powder air-abrasion [Gly]; 4)D-Limonene chemical solvent [Or]. A new IDS layer was then created; polyethylene tubes were placed on dentin surfaces and filled with a dual-cure resin cement. A universal testing machine was used for the microshear bond strength ( $\mu \mathrm{SBS}$ ) tests; conditioned surfaces were analyzed at SEM. Means and SD were calculated; a two-way ANOVA (a:0,05) was carried out to detect significant differences among groups.

For NE groups, mean $\mu \mathrm{SBS}$ values (MPa) were: 21.6 \pm 6.6 [NE_NoT]; 20.7 \pm 4.5 [NE_Or]; 20.1 $\pm 6.6\left[\mathrm{NE} \_\mathrm{SB}\right] ; 19.1 \pm 5.3$ [NE_S]; 17.8 \pm 2.2 [NE_Gly]. No significant differences were found among tested treatments within NE (p: 0,5493). For CL

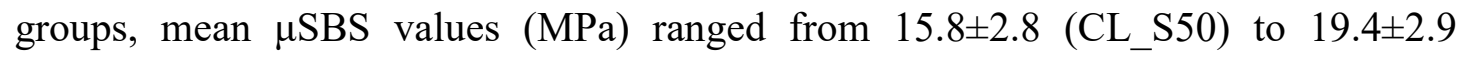
(CL_Gly). Cleaning of the substrate with glycine air-abrasion statistically improved $\mu \mathrm{SBS}$ values with respect to aluminum-oxide (CL_SB: 15.8 \pm 2.8 ) or scaling (CL_S: $16.0 \pm 2.4)$.

The application of the IDS technique was effective for preservation of freshly-cut dentin from adverse effects of temporary materials. Glycine powder air-abrasion is suggested when a temporary resin cement is adopted. 


\section{Introduction}

A wide number of tooth preparations performed in prosthetic dentistry might lead to major or minor areas of dentinal exposure $[1,2]$; the preservation of tooth's substrate with interim restorations - or at least with temporary materials - is often necessary meanwhile the laboratory workflow is carried out, also when digital productions are chosen in conjunction with outside milling centers [3].

Unfortunately, an adverse influence of commonly used temporary materials has been demonstrated on the subsequent adhesive procedures for the luting of bonded indirect restorations $[4,5]$. For example, provisional cements or sealing agents containing eugenol (a chemical component used as a catalyst) are known to reduce bond strength of definitive restorations to previously contaminated substrates [4]. Eugenol has an high-diffusion capacity into dentin, and relatively small amounts might produce interferences with contemporary adhesive techniques [6]. Moreover, the use of eugenol-free temporary materials was also found to reduce bond strength to dentin, when compared to fresh or surface-treated substrates [7]; the negative effect may not be caused by eugenol itself but by the presence of residual particles of cement [8].

An effective post-contamination cleaning of dentinal surfaces and/or prevention of substrate contamination itself are two basic approaches to reduce the negative influence of temporary materials on adhesive procedures. Adopted cleaning techniques for the removal of provisional cements range from the use of simple mechanical, hand-driven instruments (i.e: explorer, rotating brushes or burs) to more complex surface treatments like air-particle abrasion using different kinds of particles/powders [9]. Ultrasound technology has been tested for the in-vitro removal of temporary materials both from dentin and the intaglio surface of artificial crowns $[5,10]$. Er:YAG laser-irradiation was found as effective as other mechanical methods (dental probe, pumice, and cleaning bur) for the removal of a zinc-oxide provisional agent [11]; a specific type of Erbium laser, on the other hand, successfully contributed to displacement of resin cement residues before re-luting procedures [12]. In a study by Erkut et al., the effect of chemical agents (i.e: alcohol and a desiccating component) for cleaning dentin surfaces was investigated; however, according to the authors, highest bond strength were achieved with the microairborne-particle abrasion treatment [13]. 
The technique of Immediate Dentin Sealing (IDS) has been extensively studied since its proposal by Paul and Scharer in 1997 [14]; this procedure is based on the immediate application and polymerization of a dentin-bonding agent (DBA) over exposed, freshly-cut dentinal areas following tooth preparation, just before the impression recording [14]. Several advantages have been described for the IDS technique, including reduction of bacterial contamination and decreased tooth sensitivity during the provisionalization phase [15]; moreover, an improved bond strength of the final restoration with respect to a delayed dentin hybridization approach (i.e: adhesive procedures accomplished exclusively at the luting appointment) has been reported [14, 16, 17]. In fact, unsealed dentinal surfaces exposed to the oral environment (i.e: salivary fluids, bacteria, thermal stresses) or to temporary materials are thought to be a less-than-optimal substrate for delayed adhesion of bonded indirect restorations [17].

However, the behavior of dentin sealed surfaces contaminated by provisional cements seems to be more problematic. According to a study of Sailer et al., contamination of the sealed dentin substrate with a temporary, eugenol-free cement - subsequently removed with a prophylaxis paste - decreased bond strength values of a conventional resin luting agent with respect to the values obtained on sealed, not-contaminated dentin surface [18]. Ozcan et al. proposed different cleansing protocols to manage sealed dentin subsequently contaminated by a provisional cement; no significant difference was found in bond values among tested treatments, but none of them was able to reproduce bond strengths similar to a not-contaminated, not-cleaned control group (freshly ground dentin) [19]. Some conditioning treatments (i.e: like a particle abrasion with calcium carbonate, as reported by Falkensammer 2014 et al.) might also be contraindicated for the removal of temporary remnants and/or to reactivate the hybridized layer before final cementation [20]. Exploring the current literature, a definitive protocol for cleaning of the immediate sealed dentin layer has not been developed. 


\section{Objective}

The aim of this study was to evaluate the microshear bond strength of a composite resin cement to a pre-hybridized dentinal substrate exposed to two kinds of temporary materials; the influence of several chemo-mechanical techniques - adopted for the removal of temporary agents - on the adhesion to previously sealed surfaces was investigated. 


\section{Materials and Methods}

\section{Specimen preparation}

Twenty-four extracted, caries-free human third molars were collected after obtaining informed consent from the patients and used for the purposes of the study. Flat superficial dentin surfaces were created after removal of the occlusal enamel with a low-speed diamond saw (Isomet 1000, Buehler, US) under water-cooling; the enamelfree exposed dentin surfaces were further polished on wet 600,800 and 1000-grit SiC abrasive papers (10s each, in sequence) in order to standardize the smear layer. The specimens were then ultra- sonically cleaned in distilled water for 5 minutes prior to the bonding procedure to remove any remaining silicon carbide dust particles. A complete list of materials used in the study for subsequent adhesive procedures, provisional cements contamination and luting is reported in Table 1 (Table 1). One single researcher carried out all experimental steps.

\section{Procedures for Immediate Dentin Sealing (IDS)}

The adhesive procedures for development of an Immediate Dentin Sealing layer were performed on all teeth using a three-step, etch-and-rinse, light-cure bonding system (OptiBond FL,Kerr, Orange, CA) according to manufacturers' recommendations: prepared dentinal surfaces were etched with phosphoric acid (37\% H3PO4) for $15 \mathrm{~s}$, rinsed for another $15 \mathrm{~s}$ and air-dried for $5 \mathrm{~s}$. The priming agent was applied with a micro brush for $15 \mathrm{~s}$ and gently air-dried for $5 \mathrm{~s}$; subsequently, the adhesive resin was added with a brush for $15 \mathrm{~s}$ and air-thinned for $3 \mathrm{~s}$. Finally, photo-polymerization was accomplished with an irradiation of $30 \mathrm{~s}$ at $1100 \mathrm{~mW} / \mathrm{cm} 2$ using a LED polymerization unit (Bluephase C8, Ivoclar Vivadent). The obtained IDS layer on each tooth was divided into four quadrants by drawing two lines in a mesio-distal and bucco-lingual directions; one randomized quadrant was always used as a control surface (NoT), where no temporary cement and no further surface treatment were applied (i.e: noncontaminated, non-cleansed areas). The teeth were subsequently divided according to the type of temporary material used for contamination of the hybridized surfaces of the remaining three quadrants: 14 teeth were allocated to the group (NE Group) where an eugenol-free temporary cement (TempBond NE®, Kerr, Orange, CA) was used; 
the remaining 10 teeth where addressed for the experimental procedures with a resinbased temporary luting agent (TempBond Clear ${ }^{\circledR}$, Kerr, Orange, CA) (CL Group). Dentin surfaces were then coated with the provisional cement under a glass slab and stored in distilled water at $37^{\circ} \mathrm{C}$ for $24 \mathrm{~h}$.

\section{Surface treatments for removal of provisional cements}

After storage, the temporary cement layer was removed from each quadrant by using one of the following cleansing methods:

- Subgroup S (Scaler, n= 10 quadrants): an hand-scaler was used with very close, mostly overlapping parallel strokes under moderate pressure (Carvalho 2007; Junior 2010) until the dentin surfaces were visually, macroscopically free of material.

- Subgroup SB (SandBlasting, $\mathrm{n}=10$ quadrants): airborne particle abrasion using $50 \mu \mathrm{m}$ aluminum oxide particles (A12O3) applied perpendicularly to the dentin surface in rotating motions (exposure time: 20s; working distance: $10 \mathrm{~mm}$; blasting pressure: $2.8 \mathrm{bar} / 0.28 \mathrm{MPa})$.

- Subgroup Gly (Glycine Air Abrasion, $n=10$ quadrants): airborne particle abrasion using $25 \mu \mathrm{m}$ glycine powder (AirFlow Perio ${ }^{\circledR}$, EMS, Switzerland) applied perpendicularly to the dentin surface in rotating motions with the same blasting parameters reported for SB subgroup.

- Subgroup Or (Orange, $n=10$ quadrants): a liquid chemical solvent (BioOrange Solvent, Ogna S.p.a., Italy) containing D-Limonene (also known as monocyclic monoterpene) was applied with cotton pellets on the contaminated surfaces; with the aid of microbrushes the temporary cement was removed until the dentin appeared macroscopically free of debris. This treatment was exclusively reserved for samples of the NE group. It was not applied on teeth within CL Group, due to known resistance of resin-based agents to this specific solvent.

A detailed list of materials and powders used for cleansing procedures is reported in Table 2 (Table 2). For each tooth specimen, each quadrant received the cleansing treatment in a clockwise sequence. During the removal of temporary cement from one quadrant, the others three were protected with a thick polyethylene shield. 


\section{Luting procedures}

Following the application of cleansing treatments, a new IDS layer was created with same steps and materials described above; polyethylene moulds with standardized dimensions (inner surface area of $1 \mathrm{~mm} 2$; height: $4 \mathrm{~mm}$ ) were used to obtain a defined and reproducible bonding area for each quadrant. The polyethylene tubes were placed on dentin surfaces and filled with a dual-cure resin composite luting agent (NX3 Nexus ${ }^{\circledR}$, Kerr, Orange, CA); the cement was condensed with a total of two increments, and each of them was light-cured for $40 \mathrm{~s}$ at $1100 \mathrm{~mW} / \mathrm{cm} 2$ (Bluephase C8, Ivoclar Vivadent). The first increment allowed the fixation of tube on the tooth's quadrant; just before photo-polymerization, any excess of resin extending beyond the base of the vinyl-tube was removed with the help of a blade and/or microbrushes. A total of 4 bonded specimens - corresponding to the 4 quadrants - were finally obtained for each tooth. The power density of the curing device was regularly checked with a curing radiometer (Demetron, Orange, CA, USA). The specimens were then stored in distilled water for $24 \mathrm{~h}$ at $37{ }^{\circ} \mathrm{C}$ until experiments.

\section{MicroShear Bond Strength ( $\mu S B S)$ test}

A universal testing machine (Instron Testing Machine, Instron, USA) was used for the microshear bond tests. Tooth specimens were mounted in the jig and each polyethylene mould containing the bonded samples was attached to the testing device. A thin orthodontic wire for ligatures $(0.2-\mathrm{mm}$ diameter) was looped around the cylinder, embracing half its circumference, and gently held flush against the dentin at the resin-dentin interface. A shear force was applied to each specimen at a cross-head speed of $0.5 \mathrm{~mm} / \mathrm{min}$ until failure occurred. The force required to failure was then divided by the bonded area of the vinyl tube and the bond strength values expressed in MPa. Measurements were processed with appropriate software (SPSS ${ }^{2} 17$, Mac OS $\mathrm{X})$. The adhesive interface, the wire loop, and the center of the load cell were all aligned as straight as possible to ensure the correct application of the shear force.

\section{Surface topography examination}

The topographic effect of all cleansing treatments applied within NE and CL groups was analyzed by SEM (Leo 438-VP, 15 kV, Leo Electron Microscopy, UK) using three additional samples (previously hybridized, divided into quadrants and 
contaminated according to the above description); among the analyzed surfaces we included a control area made by the hybridized dentin alone (not-contaminated, not cleansed) and a layer of temporary material (TempBond NE®) left undisturbed. For SEM evaluations specimens were first marked, gold sputtered, and finally evaluated at a magnification of $\mathrm{x} 100-\mathrm{x} 1000$.

\section{Mode of failure}

Fractured interfaces of the specimens were examined with a stereomicroscope (Leica MZ12, Weitzlar, Germany) at $25 \times$ magnification to distinguish the mode of failure. Fractured sites were classified as follows: pure adhesive, in case of complete detachment of the adhesive resin from dentin; mixed, when failure occurred at the adhesive resin - dentin interface and some areas of tooth surface were still covered by the adhesive; cohesive failure within the composite cement, when fractures involved only the luting agent layer; cohesive within dentin, if the fracture occurred exclusively in dentin.

\section{Statistical Analysis}

Normal distribution of the obtained data (MPa) from $\mu$ SBS tests was checked using Kolmogorov-Smirnov evaluation; after that was confirmed, means and standard deviations were calculated. A two-way analysis of variance (two-way ANOVA) was carried out (taking into account the main factors "Temporary luting agent" and "Cleansing Method" on shear bond values) in order to detect potential significant differences among study groups. Statistical significance was set at $5 \%(\mathrm{P}=0.05)$. 


\section{Results}

Table 3 shows means and standard deviations of the $\mu$ SBS testing, for the two tested temporary agents. For NE Group, mean $\mu \mathrm{SBS}$ values (MPa) ranged from $17.8 \pm 2.2$ (NE_Gly) to $21.6 \pm 6.6$ (NE_NoT); no significant differences were found among tested surface treatments (p: 0.5493). Best cleaning method within the NE ranking was obtained with chemical conditioning using D-Limonene (NE_Or), with $\mu$ SBS values $(20.7 \pm 4.5)$ closely approaching those of NE_NoT.

For CL Group, mean $\mu$ SBS values (MPa)ranged from $15.8 \pm 2.8$ (CL_S50) to $19.4 \pm$ 2.9 (CL_Gly). A significant difference was found among study groups of the temporary resin-based luting agent (p: 0.0188): cleaning of the substrate with Glycine air-abrasion statistically improved $\mu$ SBS values with respect to aluminum-oxide sandblasting (CL_SB: $15.8 \pm 2.8)$ or scaling (CL_S: $16.0 \pm 2.4)$.

Failure types were predominantly mixed (i.e: between the dentin and the adhesive resin); cohesive failures in the dentin were not observed in any of the groups. According to the two-way analysis of variance, no significant interaction was found among considered factors "Temporary luting agent" and "Cleansing Method" on shear bond strength values (Treatment * Temp_Cem; $\mathrm{p}=0.1063$ ).

Figure 1 and Figure 2 show representative SEM images of specimen surfaces for NE and CL groups, respectively. For each picture, low (100x) and high (1000x) magnifications are reported with additional caption " 1 " or " 2 ", respectively. Typical topographic features were found for S and SB cleaning methods, regardless the temporary agent applied: pictures revealed an increased surface roughness with grooves and micro-cracks formation for alumina abrasion (see Fig. 1C1-1C2 and Fig. 2B1-2B2); partial cement removal and scratches from the scaler blade were found for Ne_S (Fig. 1B1-1B2) and CL_S (Fig.2A1-2A2) groups. More heterogeneous surfaces were highlighted for Gly treatments (Fig. 1D1), with areas of increased roughness (Fig. 1D2; Fig. 2C1), partial tubule openings and/or persistence of adhesive remnants (Fig. 2C2). AT SEM analysis, some amount of debris were always present, regardless the cleaning technique. 


\section{Discussion}

The clinical, long-term success of bonded indirect restorations depends, among other factors, on reliable adhesion between prosthetic materials and tooth's tissues [21]; for this reason, a deep or complete removal of temporary materials is required to achieve cleaned dentinal surfaces before luting of permanent restorations. The Immediate Dentin Sealing (IDS) is a technique to preserve freshly cut dentin - considered an ideal substrate for adhesive procedures - from the potential, negative effects of temporary materials [14]. In other words, adhesion values to tooth's surfaces treated by the IDS approach and exposed to temporary materials should be comparable to values obtained on hybridized but not contaminated dental surfaces.

Several authors have supported the IDS technique for cementation of indirect restorations $[14,16,17,22]$; in particular, most updated reviews suggest its advantages over a delayed cementation protocol [15, 23]. Magne et al. have demonstrated it was possible to obtain an efficient bond between the resin-coated dentin and the final restoration after 2 to 4 months of placement of provisional materials; in their study, the authors reported high microtensile bond strength values ( $>45 \mathrm{MPa}$ ) within the IDS groups that were similar to those obtained using a freshly placed adhesive [22]. Magne et al. recommended and applied a micro-airborne particle abrasion $\left(30 \mu \mathrm{m}\right.$ aluminum oxide powder, Cojet ${ }^{\circledR}$ System, 3M ESPE) for cleaning of dentin/removal of temporary material [22]. According to Dillenburg et al. the use of aluminum-oxide air-abrasion for the removal of temporary cement from IDS surfaces was effective to produce bond values similar to the control groups (sealed but uncontaminated substrates) [24].

In our study, both mechanical (S, SB, and Gly) and chemical (Or) treatments (that represent common chair-side instrument and techniques adopted by clinicians) were effective for the removal of provisional cements when looking at adhesive results: in fact, no significant differences were found between conditioned and control groups, regardless the type of temporary agent (TempBond NE® or TempBond Clear ${ }^{\circledR}$ ). Within CL Group, however, $\mu$ SBS values were generally lower, probably highlighting a more difficult removal of particles of the resin-based, light-cured luting agent. Altintas et al. also reported lower bond values for TempBond Clear® compared to other types of temporary cements (Eugenol-free or calcium hydroxide), regardless the 
cleaning procedure; according to the authors, few resin tags formation were revealed by SEM, probably attributed to the presence of provisional cement residues. From a clinical standpoint, intrinsic transparency of the tested resin-based cement, or different detachment features with respect to TempBond®NE, might increase difficulty of removal from tooth surface.

For both CL and NE groups, the removal of temporary cements with an hand instrument (i.e: scaler) produced bond values $(\mathrm{MPa})$ comparable to those obtained with $50 \mu \mathrm{m}$ sandblasting treatment (CL_S50:15.8 Vs CL_S:16.0; NE_S50:20.1 Vs NE_S:19.1): although some cleaning procedures may appear less predictable for decontamination, curettes/hand excavators or prophylaxis paste/pumice slurry resulted to be clinically effective. In fact, according to Ozcan et al. no difference on adhesion values was found between the removal of temporary cements with a prophylaxis paste or with two different sandblasting regimens (2 or 3.5 bar pressure) [19]. Abo-Hamar et al. also identified a comparable efficacy between cleanings with sandblasting and curettes [25]. On the other hand, it should be emphasized that a prolonged, overextended action of some surface treatments might also produce obscuration of dentinal tubule openings due to formation of additional smear layers, finally providing less reliable adhesion results [26]. That problem is also observed with other more aggressive mechanical methods involving finishing or tungsten carbide burs, that might impair precise fitting of indirect restorations [19]; for this reason, they are not suggested for surface cleanings, and where not included in the present study. One previous research have investigated the effect of mechanical treatments (i.e: polishing and air abrasion) on IDS layers [27]: as revealed by SEM analysis, the amount of hybridized layer that was removed with that cleaning methods was not uniform (11.94 $+/-16.46 \mu \mathrm{m})$, and a great range of values was recorded (0 to $145 \mu \mathrm{m})$; however, in the majority of cases, a minimum thickness of adhesive layer was preserved. This fact could be particularly beneficial for subsequent adhesion, since development of covalent bonding between the adhesive resin (with its available free monomers) and the methacrylate groups of the resin luting cement is possible [19, 28].

Looking at adhesion values, the range of our results was placed midway between the studies of Carvalho et al. (19.7 - 31.3 MPa) and those of Ozcan et al. (5.2-8.0 MPa); the adoption of different adhesive systems and temporary cement in one case [29], and of different treatments in the other [19], might explain the obtained differences. 
However, the same testing method was employed: the microshear technique reduces both cohesive failures of the substrate observed in macro-shear evaluations [30], and it avoids potential pre-test failures or misalignment problems encountered in microtensile tests [31]. Among the advantages of microshear method, a more accurate measurement of bonding values is possible due to specific concentration of stresses between adherent and substrate interface $[31,32]$.

Despite an overall efficacy of tested treatments for obtaining satisfactory $\mu$ SBS values, most SEM images revealed a persistence of cement residues on dentin surfaces; in other words, none of our proposed conditionings was actually capable of completely removing all particles of the temporary agent. Other surface treatments or a combination of them - to be tested in future investigations - might additionally increase the adhesion results by an optimized cleaning of the IDS layer. Heterogeneous but mostly clean surfaces, with a minor partial exposure of dentinal tubuli, were highlighted when Glycine air-abrasion was applied for the removal of TempBond ${ }^{\circledR}$ Clear (see Fig. $2 \mathrm{C} 1$ and Fig. 2C2); compared to $50 \mu \mathrm{m}$ alumina sandblasting which produced several microcracks or grooves on dentin, an overall less-aggressive abrasion pattern was found for glycine treatment. We may speculate that the above-mentioned microscopic features might explain the enhanced results obtained for CL_Gly Group with respect to CL_S and Cl_SB.

Beside the in-vitro nature of this study, which facilitated the application of a cleansing method with respect to a clinical scenario, other limitations were present; a longer period of storage of the samples (contaminated with the temporary agent), or aging of the bonded interfaces with thermal cycles - to be explored in future studies - may bring additional information on the adhesion behaviour of resin composite cements to IDS layers. 


\section{Conclusions}

The application of the immediate dentin sealing (IDS) technique, in association with one of the tested cleaning methods, was effective for preservation of freshly-cut dentin from adverse effects of temporary materials. Best $\mu$ SBS values were identified for specific temporary luting-agent/surface treatment combinations; glycine airabrasion was particularly effective for the removal of the resin-based, light-cure temporary cement.

\section{Acknowledgements}

Special thanks to Dott. Matteo Bosisio for his kind support and to the Kerr ${ }^{\mathrm{TM}}$ Group for the materials provided in this research. 


\section{Legend}

Tab. 1 - The brands, chemical compositions, manufacturers and batch numbers of the materials used for the experiments.

Tab. 2 - Materials used during surface treatments for removal of temporary agents.

Tab. 3 - Microshear ( $\mu$ SBS) bond strengths (means, standard deviations and minimummaximum intervals expressed as MPa) of resin composite cement on dentin after cleansing methods.

Figure 1 - Appearance of an immediately sealed dentin layer (A1-A2) and surfaces following

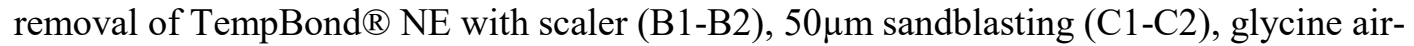
abrasion (D1, D2), or chemical solvent (E1-E2).

\section{Sublegends for Figure 1}

Figure 1 A1: Uniform, plain appearance of adhesive layer at 100x (NoT group).

Figure 1 A2: The IDS surface at higher magnification (1000x) shows some voids and gaps, along with some little impurities on the right side.

Figure 1 B1: Dentinal surface following removal of temporary material with a scaler (S Group): the blade produced long, multi-directional scratches; different contact angles with dentin explain variations in size/widths of the carvings. Minor and major debris are visible. $100 x$.

Figure 1 B2: Beside debris, both partially occluded and open dentinal tubules are present; in some areas, it seems the scaler has removed the superficial adhesive layer. 1000x.

Figure 1 C1: After treatment with 50 $\mu \mathrm{m}$ alumina sandblasting (NE_S50 Group) an increased surface roughness is noted; the abraded area appeared homogeneous and mostly free of debris. 100x.

Figure 1 C2: Multiple grooves are developed from the impact of alumina particles with the surface; the dentinal structure or its tubules are not recognized. 1000x.

Figure 1 D1: The removal of TempBond NE® with glycine powder produced an heterogeneous, irregular dentinal surface (NE_Gly); the persistence of an adhesive residue is suggested by the dark island (left side) surrounded by some exposed tubuli. 100x. Figure 1 D2: Increased surface roughness is obtained with glycine air abrasion; however, $25 \mu \mathrm{m}$ particles produced little or no micro-cracks with respect to alumina sandblasting. $1000 \mathrm{x}$.

Figure $1 \mathbf{E 1}$ : The surface is crossed with signs related to cotton pellets, brushes and tweezers tips used for cleaning of the surface with the liquid solvent (NE Or Group). Some areas appear cleaner than others producing a chalkboard-like surface. $100 x$.

Figure 1 E2: Uneven dissolution of temporary agent by the chemical solvent; some debris might be transported with cotton pellets during cleaning procedures. 1000x. 
Figure 2 - Appearance of dentinal surfaces following removal of TempBond ${ }^{\circledR}$ Clear with scaler (A1-A2), 50 $\mu \mathrm{m}$ sandblasting (B1-B2) and glycine air-abrasion (C1, C2).

\section{Sublegends for Figure 2}

Figure 2 A1: Resin-based temporary agent removed with scaler (CL_S Group): longitudinal scratches are visible and the dentin surface is still covered by some residual fragments of cement. 100x.

Figure 2 A2: Detail (1000x) of a fractured layer of temporary cement; dentin has been exposed by the scaler only in the upper left part of the image.

Figure 2 B1: Regardless the temporary agent a standardized, specific surface feature was obtained with alumina sandblasting (see Fig. 1C1-1C2). 100x.

Figure 2 B2: A compact, amorphous surface with several micro-cracks is observed at 1000x magnification.

Figure 2 C1: A central area of parallel tracks with exposed dentinal tubuli is visible; right and left zones are crossed by thin, shallow micro cracks. 100x.

Figure 2 C2: At high magnification the surface is almost clean and homogeneous; the underlying structure of dentin is identified with few open dentinal tubuli. 1000x. 
Preview of Figure 1 (low-size and reduced pictures for illustration purposes only).

Appearance of an immediately sealed dentin layer (A1-A2) and surfaces following removal of TempBond $\circledast$ NE with scaler (B1-B2), 50 $\mu \mathrm{m}$ sandblasting (C1-C2), glycine air-abrasion (D1,

D2), or chemical solvent (E1-E2).

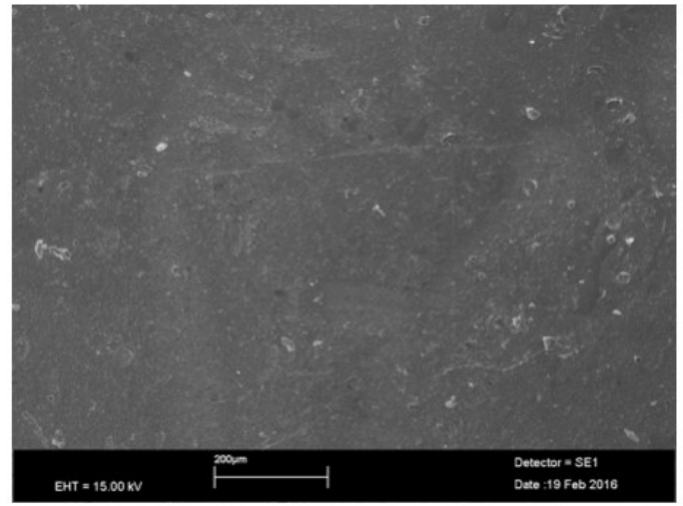

A1: Uniform, plain appearance of adhesive layer at 100x (NoT group).

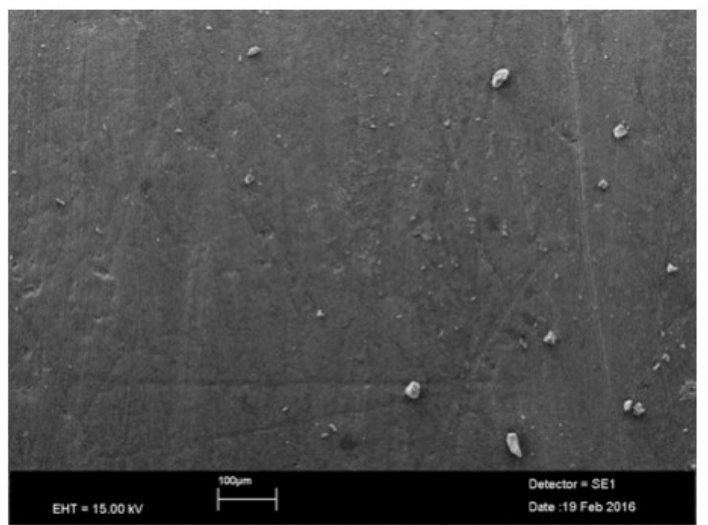

B1: Dentinal surface following removal of temporary material with a scaler (S Group): the blade produced long, multidirectional scratches; different contact angles with dentin explain variations in size/widths of the carvings. Minor and major debris are visible. 100x.

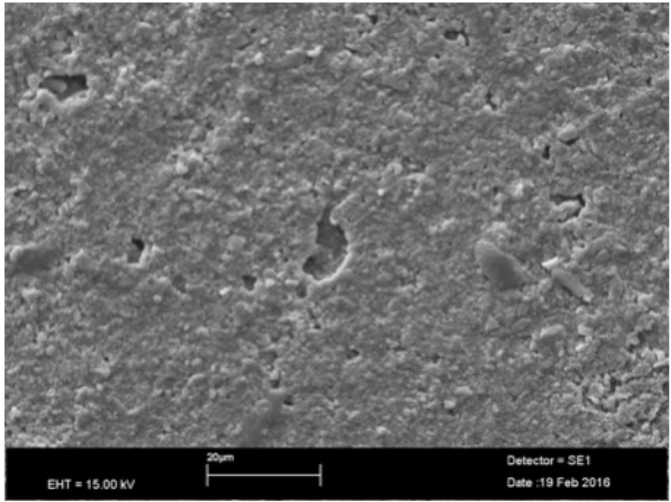

A2: The IDS surface at higher magnification (1000x) shows some voids and gaps, along with some little impurities on the right side.

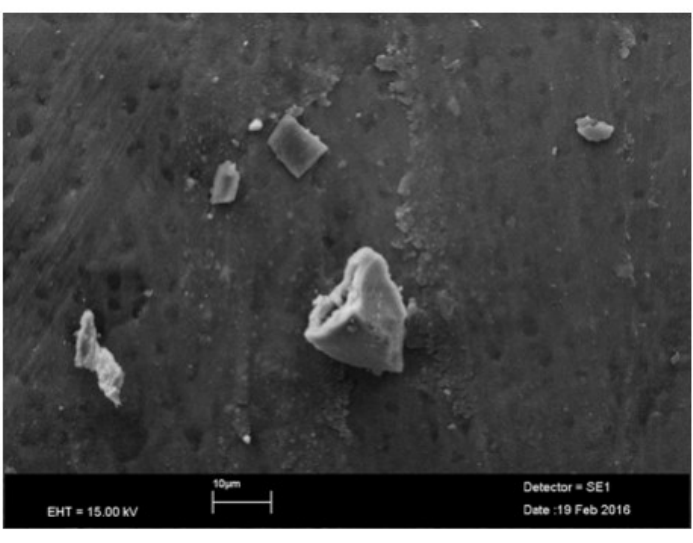

B2: Beside debris, both partially occluded and open dentinal tubules are present; in some areas, it seems the scaler has removed the superficial adhesive layer. 1000x. 


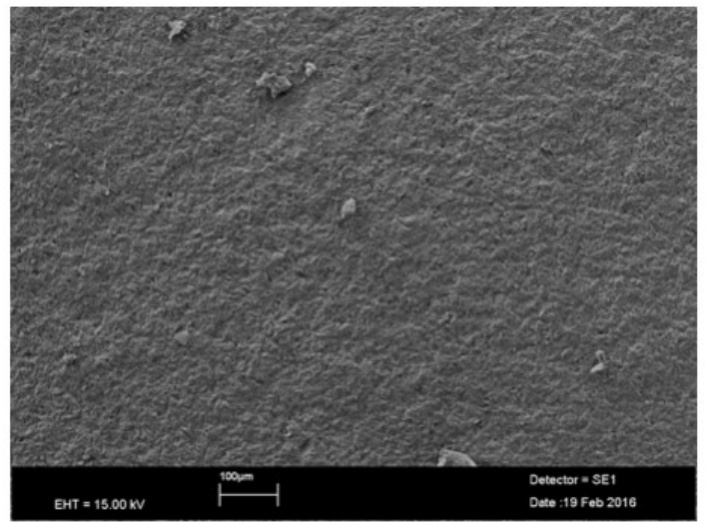

C1: After treatment with $50 \mu \mathrm{m}$ alumina sandblasting (NE_S50 Group) an increased surface roughness is noted; the abraded area appeared homogeneous and mostly free of debris. 100x.

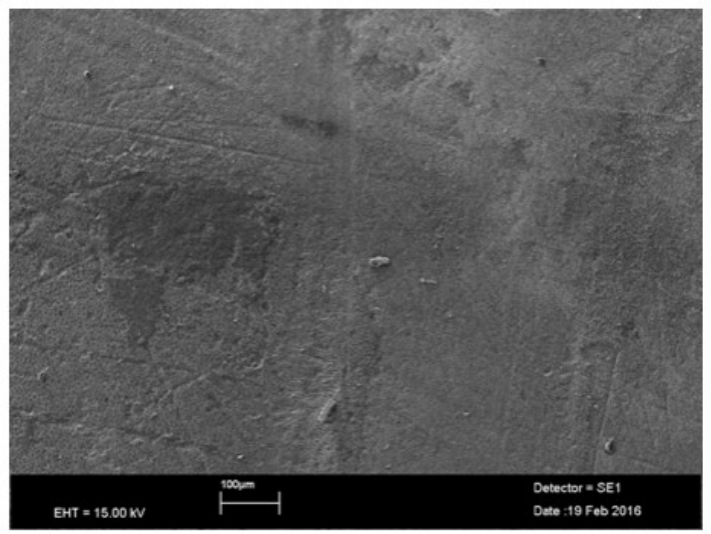

D1: The removal of TempBond NE® with glycine powde produced an heterogeneous, irregular dentinal surface
(NE Gly); the persistence of an adhesive residue is

(NE_.Gly); the persistence of an adhesive residue is exposed tubuli. 100x.

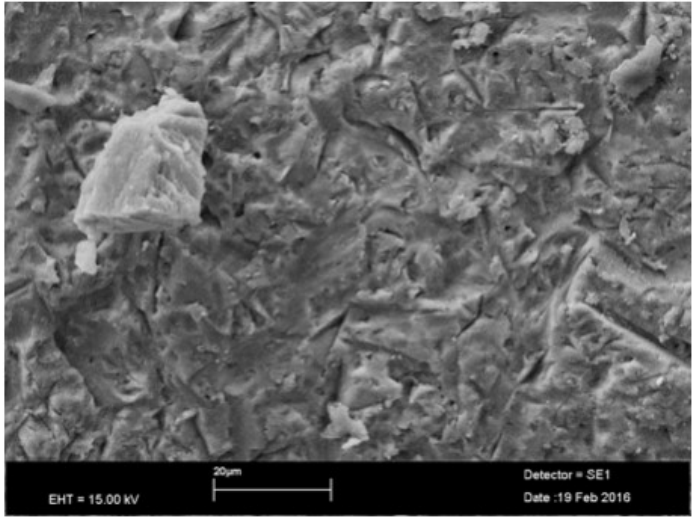

C2: Multiple grooves are developed from the impact of alumina particles with the surface; the dentinal structure or its tubules are not recognized. 1000x.

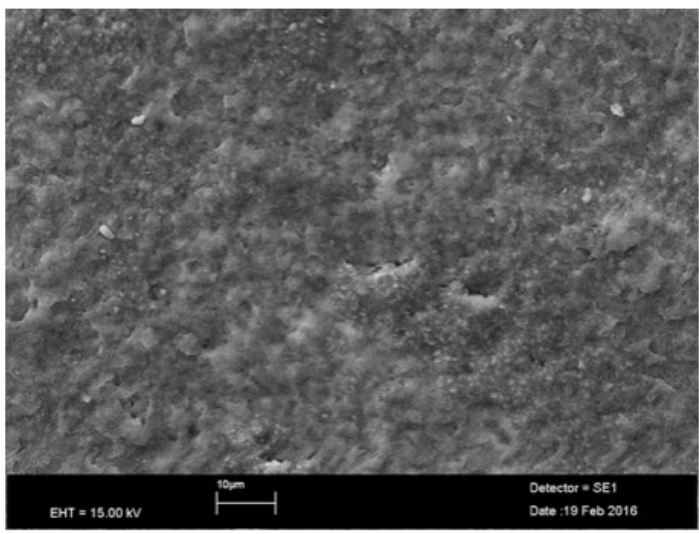

D2: Increased surface roughness is obtained with glycine air abrasion; however, $25 \mu \mathrm{m}$ particles produced little or no micro-cracks with respect to alumina sandblasting. $1000 \mathrm{x}$. 

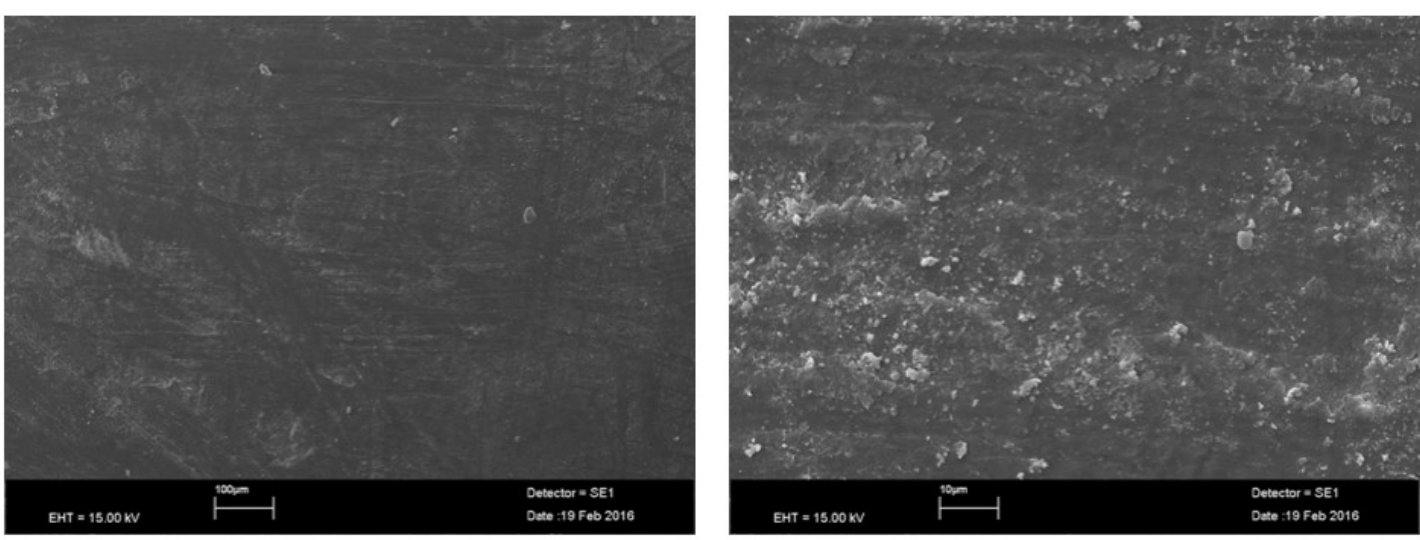

E1: The surface is crossed with signs related to cotton pellets, brushes and tweezers tips used for cleaning of the surface with the liquid solvent (NE_Or Group). Some area appear cleaner than others producing a chalkboard-like

E2: Uneven dissolution of temporary agent by the chemical solvent; some debris might be transported with cotton pellets during cleaning procedures. $1000 \mathrm{x}$. surface. 100x. 
Preview of Figure 2 (low-size and reduced pictures for illustration purposes only).

Appearance of dentinal surfaces following removal of TempBond@ Clear with scaler (A1-A2), $50 \mu \mathrm{m}$ sandblasting (B1-B2) and glycine air-abrasion (C1, C2).
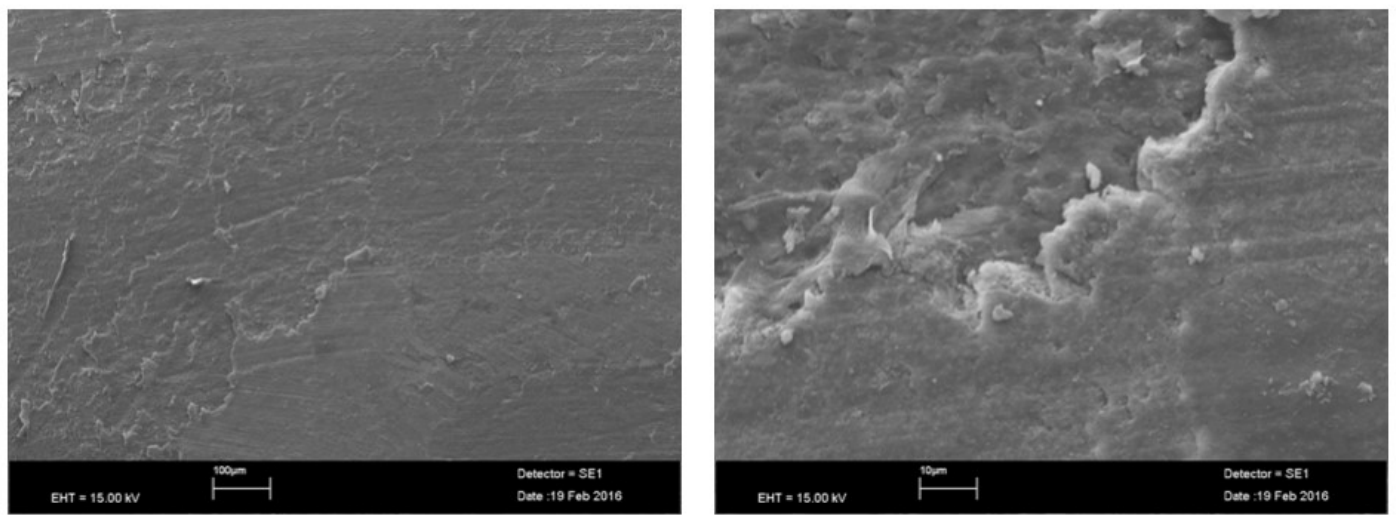

A1: Resin-based temporary agent removed with scaler

(CL_S Group).: longitudinal scratches are visible (as shown in Fig. 2B) and the dentin surface is still covered by some residual

A2: Detail (1000x) of a fractured layer of temporary cement; dentin has been exposed by the scaler only in the upper left part of the image.

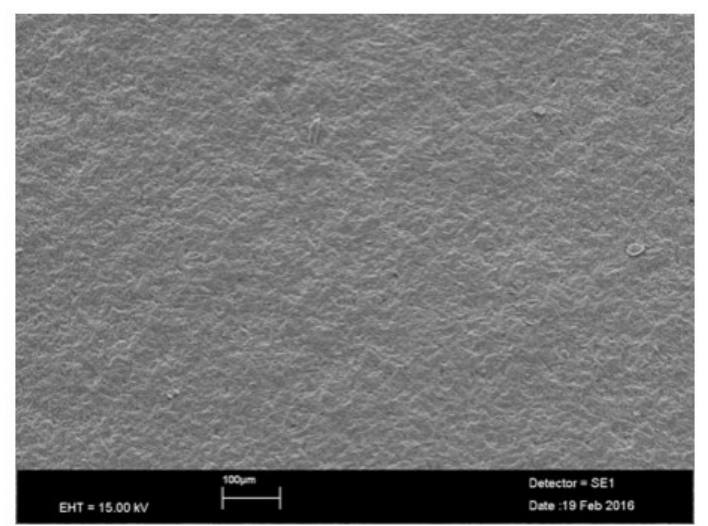

B1: Regardless the temporary agent a standardized, specific surface feature was obtained with alumina sandblasting (see Fig. 2C). $100 x$.

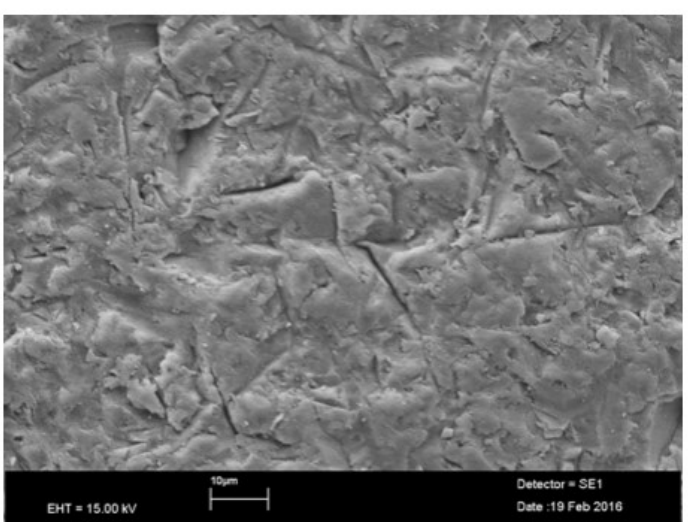

B2: A compact, amorphous surface with several micro-cracks is observed at $1000 x$ magnification. 

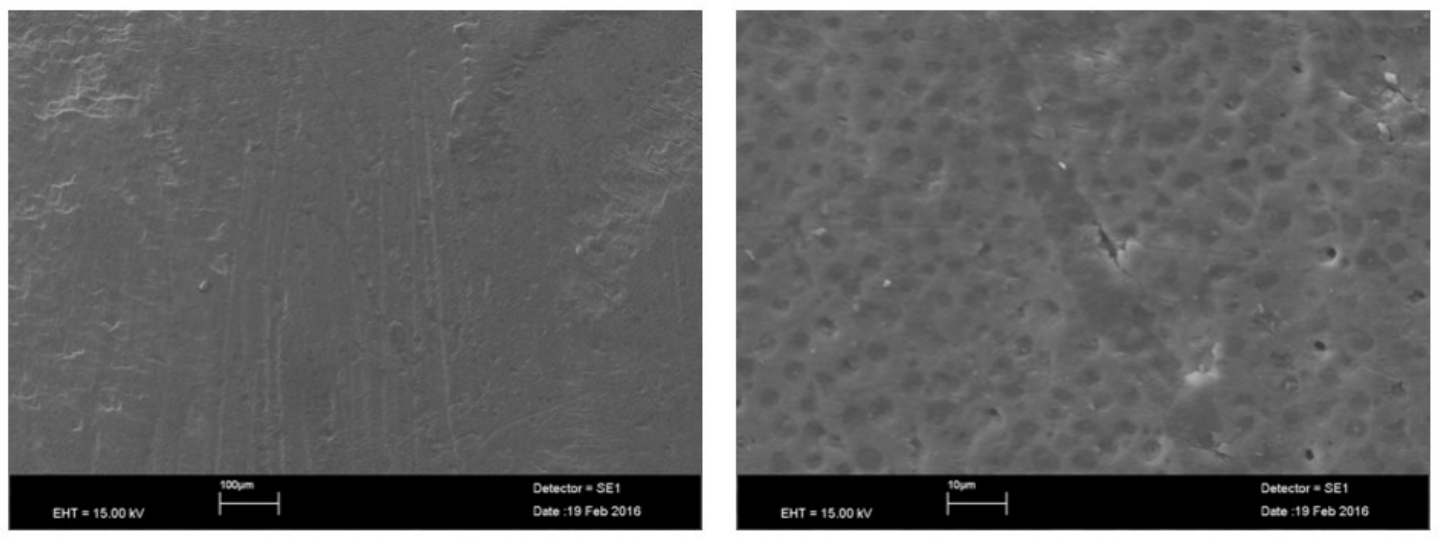

C1: A central area of parallel tracks with exposed dentinal tubuli is visible; right and left zones are crossed by thin, shallow micro

C2: At high magnification the surface is almost clean and homogeneous; the underlying structure of dentin is identified with cracks. 100x. 


\section{References}

1. Borelli B, Sorrentino R, Goracci C, Amato M, Zarone F, Ferrari M. Evaluating residual dentin thickness following various mandibular anterior tooth preparations for zirconia fullcoverage single crowns: an in vitro analysis. Int J Periodontics Restorative Dent. 2015;35:417.

2. Ozturk E, Bolay S. Survival of porcelain laminate veneers with different degrees of dentin exposure: 2-year clinical results. J Adhes Dent. 2014;16:481-9.

3. Tordiglione L, De Franco M, Bosetti G. The Prosthetic Workflow in the Digital Era. Int J Dent. 2016;2016:9823025.

4. Ribeiro JC, Coelho PG, Janal MN, Silva NR, Monteiro AJ, Fernandes CA. The influence of temporary cements on dental adhesive systems for luting cementation. J Dent. 2011;39:25562.

5. Takimoto M, Ishii R, lino M, Shimizu Y, Tsujimoto A, Takamizawa T, Ando S, Miyazaki M. Influence of temporary cement contamination on the surface free energy and dentine bond strength of self-adhesive cements. J Dent. 2012;40:131-8.

6. Fiori-Junior M, Matsumoto W, Silva RA, Porto-Neto ST, Silva JM. Effect of temporary cements on the shear bond strength of luting cements. J Appl Oral Sci. 2010;18:30-6.

7. Carvalho EM, Carvalho CN, Loguercio AD, Lima DM, Bauer J. Effect of temporary cements on the microtensile bond strength of self-etching and self-adhesive resin cement. Acta Odontol Scand. 2014;72:762-9.

8. Watanabe EK, Yamashita A, Imai M, Yatani H, Suzuki K. Temporary cement remnants as an adhesion inhibiting factor in the interface between resin cements and bovine dentin. Int J Prosthodont. 1997;10:440-52.

9. Chaiyabutr $Y$, Kois JC. The effects of tooth preparation cleansing protocols on the bond strength of self-adhesive resin luting cement to contaminated dentin. Oper Dent. 2008;33:556-63.

10. Mosharraf R, Soleimani B, Sanaee-Nasab M. A comparison of two methods of removing zinc oxide-eugenol provisional cement residue from the internal surface of cast restorations. J Contemp Dent Pract. 2009;10:27-34.

11. Zortuk M, Gumus HO, Kilinc HI, Tuncdemir AR. Effect of different provisional cement remnant cleaning procedures including Er:YAG laser on shear bond strength of ceramics. J Adv Prosthodont. 2012;4:192-6.

12. Tasar S, Ulusoy MM, Meric G. Microshear bond strength according to dentin cleansing methods before recementation. J Adv Prosthodont. 2014;6:79-87.

13. Erkut S, Yilmaz B, Bagis B, Kucukesmen C, Ozdemir E, Acar O. Effect of different surfacecleaning techniques on the bond strength of composite resin restorations. J Prosthet Dent. 2014;112:949-56. 
14. Paul SJ, Scharer P. The dual bonding technique: a modified method to improve adhesive luting procedures. Int J Periodontics Restorative Dent. 1997;17:536-45.

15. Qanungo A, Aras MA, Chitre V, Mysore A, Amin B, Daswani SR. Immediate dentin sealing for indirect bonded restorations. J Prosthodont Res. 2016;60:240-9.

16. Choi YS, Cho IH. An effect of immediate dentin sealing on the shear bond strength of resin cement to porcelain restoration. J Adv Prosthodont. 2010;2:39-45.

17. Magne P. Immediate dentin sealing: a fundamental procedure for indirect bonded restorations. J Esthet Restor Dent. 2005;17:144-54; discussion 55.

18. Sailer I, Oendra AE, Stawarczyk B, Hammerle CH. The effects of desensitizing resin, resin sealing, and provisional cement on the bond strength of dentin luted with self-adhesive and conventional resincements. J Prosthet Dent. 2012;107:252-60.

19. Özcan M, Lamperti S. Effect of mechanical and air-particle cleansing protocols of provisional cement on immediate dentin sealing layer and subsequent adhesion of resin composite cement. Journal of Adhesion Science and Technology. 2015;29:2731-43.

20. Falkensammer F, Arnetzl GV, Wildburger A, Krall C, Freudenthaler J. Influence of different conditioning methods on immediate and delayed dentin sealing. J Prosthet Dent. 2014;112:204-10.

21. Burke FJ, Fleming GJ, Nathanson D, Marquis PM. Are adhesive technologies needed to support ceramics? An assessment of the current evidence. J Adhes Dent. 2002;4:7-22.

22. Magne $P$, So WS, Cascione D. Immediate dentin sealing supports delayed restoration placement. J Prosthet Dent. 2007;98:166-74.

23. Nawareg MM, Zidan AZ, Zhou J, Chiba A, Tagami J, Pashley DH. Adhesive sealing of dentin surfaces in vitro: A review. Am J Dent. 2015;28:321-32.

24. Dillenburg AL, Soares CG, Paranhos MP, Spohr AM, Loguercio AD, Burnett LH, Jr. Microtensile bond strength of prehybridized dentin: storage time and surface treatment effects. J Adhes Dent. 2009;11:231-7.

25. Abo-Hamar SE, Federlin M, Hiller KA, Friedl KH, Schmalz G. Effect of temporary cements on the bond strength of ceramic luted to dentin. Dent Mater. 2005;21:794-803.

26. Santos MJ, Bapoo H, Rizkalla AS, Santos GC. Effect of dentin-cleaning techniques on the shear bond strength of self-adhesive resin luting cement to dentin. Oper Dent. 2011;36:51220.

27. Stavridakis MM, Krejci I, Magne P. Immediate dentin sealing of onlay preparations: thickness of pre-cured Dentin Bonding Agent and effect of surface cleaning. Oper Dent. 2005;30:747-57.

28. Brendeke J, Ozcan M. Effect of physicochemical aging conditions on the compositecomposite repair bond strength. J Adhes Dent. 2007;9:399-406. 
29. Carvalho CN, de Oliveira Bauer JR, Loguercio AD, Reis A. Effect of ZOE temporary restoration on resin-dentin bond strength using different adhesive strategies. J Esthet Restor Dent. 2007;19:144-52; discussion 53.

30. Braga RR, Meira JB, Boaro LC, Xavier TA. Adhesion to tooth structure: a critical review of "macro" test methods. Dent Mater. 2010;26:e38-49.

31. Armstrong S, Geraldeli S, Maia R, Raposo LH, Soares CJ, Yamagawa J. Adhesion to tooth structure: a critical review of "micro" bond strength test methods. Dent Mater. 2010;26:e5062.

32. Placido E, Meira JB, Lima RG, Muench A, de Souza RM, Ballester RY. Shear versus microshear bond strength test: a finite element stress analysis. Dent Mater. 2007;23:1086-92. 